\title{
DNA damage in blood leucocytes of prostate cancer patients during therapy with ${ }^{177}$ Lu-PSMA
}

\author{
Sarah Schumann ${ }^{1}$ (I) $\cdot$ Harry Scherthan ${ }^{2} \cdot$ Constantin Lapa $^{1} \cdot$ Sebastian Serfling $^{1} \cdot$ Razan Muhtadi $^{2} \cdot$ Michael Lassmann $^{1}$. \\ Uta Eberlein ${ }^{1}$
}

Received: 23 November 2018 / Accepted: 22 March 2019 /Published online: 27 April 2019

(C) The Author(s) 2019

\begin{abstract}
Purpose The aim of this study was to investigate the time- and dose-dependency of DNA double-strand break (DSB) induction and repair in peripheral blood leucocytes of prostate cancer patients during therapy with ${ }^{177} \mathrm{Lu}$-PSMA.

Methods Blood samples from 16 prostate cancer patients receiving their first ${ }^{177}$ Lu-PSMA therapy were taken before and at seven time-points (between $1 \mathrm{~h}$ and $96 \mathrm{~h}$ ) after radionuclide administration. Absorbed doses to the blood were calculated using integrated time-activity curves of the blood and the whole-body. For DSB quantification, leucocytes were isolated, fixed in ethanol and immunostained with $\gamma-\mathrm{H} 2 \mathrm{AX}$ and 53BP1 antibodies. Colocalizing foci of both DSB markers were manually counted in a fluorescence microscope.

Results The average number of radiation-induced foci (RIF) per cell increased within the first $4 \mathrm{~h}$ after administration, followed by a decrease indicating DNA repair. The number of RIF during the first $2.6 \mathrm{~h}$ correlated linearly with the absorbed dose to the blood $\left(R^{2}=0.58\right)$, in good agreement with previously published in-vitro data. At late time-points $(48 \mathrm{~h}$ and $96 \mathrm{~h}$ after administration), the number of RIF correlated linearly with the absorbed dose rate $\left(R^{2}=0.56\right)$. In most patients, DNA DSBs were repaired effectively. However, in some patients RIF did not disappear completely even $96 \mathrm{~h}$ after administration.

Conclusion The general pattern of the time- and dose-dependent induction and disappearance of RIF during ${ }^{177}$ Lu-PSMA therapy is similar to that of other radionuclide therapies.
\end{abstract}

Keywords DNA double-strand breaks $\cdot \gamma$-H2AX $\cdot 53 \mathrm{BP} 1 \cdot$ Biodosimetry $\cdot$ Absorbed dose to the blood $\cdot$ Prostate cancer $\cdot$ ${ }^{177}$ Lu-PSMA

\section{Introduction}

Membrane glycoprotein prostate-specific membrane antigen (PSMA) is highly overexpressed in prostate cancer and metastases, making it a particularly suitable target for imaging and therapeutic agents $[1,2]$. For the last 5 years, PSMAbinding ligands labelled with the $\beta$-particle-emitter ${ }^{177} \mathrm{Lu}$ have been used in an increasing number of medical centres worldwide for the treatment of metastatic castration-resistant

Sarah Schumann

Schumann_S1@ukw.de

1 Department of Nuclear Medicine, University of Würzburg, Oberdürrbacher Str. 6, 97080 Würzburg, Germany

2 Bundeswehr Institute of Radiobiology affiliated to the University of Ulm, Neuherbergstr. 11, 80937 Munich, Germany prostate cancer. Various studies have shown promising results including high response rates, a favourable safety profile and reduction in pain [3-8]. However, the ionising radiation from ${ }^{177} \mathrm{Lu}$ not only destroys malignant tissue, but also leads to DNA damage in healthy cells. In particular, the haematopoietic system is an organ-at-risk for targeted radionuclide therapies. As the absorbed dose to the blood can be used as a surrogate marker for the absorbed dose to the bone marrow, the quantification of radiation-induced DNA damage in blood and its correlation with the absorbed dose to the blood is of great interest [9].

Among the different types of DNA damage, double-strand breaks (DSBs) are the most critical lesions, since they are difficult to repair, and misrepair can lead to mutations or cell death. DNA DSBs can be visualized by immunofluorescence staining with antibodies for $\gamma-\mathrm{H} 2 \mathrm{AX}$ and 53BP1 [10-12]. The microscopically visible colocalizing $\gamma-\mathrm{H} 2 \mathrm{AX}+53 \mathrm{BP} 1$ foci in the cell nuclei can then be quantified to assess DNA DSB 
damage. Since these foci appear with the induction of DNA DSBs and disappear with the progression of DSB repair, they can be used to describe the time-dependency of DNA damage induction and repair during irradiation [13-16].

So far, only a few studies have investigated radiationinduced DNA damage during radionuclide therapy by quantifying $\gamma-\mathrm{H} 2 \mathrm{AX}$ foci or colocalizing $\gamma-\mathrm{H} 2 \mathrm{AX}+53 \mathrm{BP} 1$ foci. Three studies have addressed focus formation during radioiodine therapy $[9,15,17]$. Two studies analysed the kinetics of focus formation during peptide receptor radionuclide therapy with ${ }^{177} \mathrm{Lu}$-labelled DOTA-D-Phe-Tyr3-octreotate $\left({ }^{177} \mathrm{Lu}\right.$-DOTATATE) and ${ }^{177} \mathrm{Lu}$-labelled DOTA-D-Phe-Tyr3octreotide $\left({ }^{177}\right.$ Lu-DOTATOC) $[14,18]$. Despite the increasing number of radionuclide therapies, DNA damage in blood leucocytes of patients during therapy with PSMA-binding ligands has not been investigated until now.

Therefore, the aim of the present study was to investigate the time- and dose-dependency of DSB induction and repair in blood leucocytes of prostate cancer patients during their first therapy cycle with ${ }^{177} \mathrm{Lu}$-PSMA.

\section{Materials and methods}

\section{Patients}

The study included 16 prostate cancer patients (P1-P16) receiving their first treatment with ${ }^{177} \mathrm{Lu}$-PSMA. A standard activity of $6 \mathrm{GBq}$ of a PSMA ligand (PSMA I\&T; SCINTOMICS GmbH, Munich, Germany) labelled with ${ }^{177} \mathrm{Lu}$ (EndolucinBeta®; ITM Isotopen Technologien München AG, Munich, Germany) was intravenously administered over $20 \mathrm{~min}$. The end of the administration process was chosen as the starting and reference point for this study. The patients were hospitalized for 2 days after the start of the treatment. All patients underwent a diagnostic PET/CT scan with a ${ }^{68} \mathrm{Ga}$-labelled PSMA ligand for uptake detection and staging/restaging of the disease and a MAG3 scan with ${ }^{99 \mathrm{~m}} \mathrm{Tc}$ before and after therapy to assess kidney function. Serum PSA levels were also determined before and after therapy. Therapy response was evaluated based on the imaging results, according to the modified PET Response Criteria in Solid Tumors (mPERCIST) and RECIST1.1 [19, 20], and changes in serum PSA levels, in analogy to the PCWG3 criteria [21].

\section{Blood sampling}

Blood samples were taken prior to administration $\left(t_{0}\right.$; for determination of the individual number of baseline foci) and nominally at $1 \mathrm{~h}\left(t_{1}\right), 2 \mathrm{~h}\left(t_{2}\right), 3 \mathrm{~h}\left(t_{3}\right), 4 \mathrm{~h}\left(t_{4}\right), 24 \mathrm{~h}\left(t_{5}\right), 48 \mathrm{~h}\left(t_{6}\right)$ and $96 \mathrm{~h}\left(t_{7}\right)$ after administration. Blood was drawn using lithiumheparin blood-collecting tubes (S-Monovette®; Sarstedt, Nümbrecht, Germany). Due to variations in the management of the individual patients, there were occasional deviations from the nominal sampling time-points. The last blood sample $(96 \mathrm{~h}$ after administration) was missing in patients $\mathrm{P} 5, \mathrm{P} 8$ and $\mathrm{P} 12$. In patient P11, the $t_{3}$ sample could not be evaluated.

\section{Activity quantification}

An aliquot of each blood sample was measured in a calibrated high-purity germanium detector (Canberra, Rüsselsheim, Germany). The counting efficiency of the detector was ascertained by measuring several NIST- and NPL-traceable standards. For activity quantification, the $\gamma$-emission line of ${ }^{177} \mathrm{Lu}$ at $208.4 \mathrm{keV}$ (emission probability of $10.4 \%$ ) was evaluated. The measured number of counts was decay-corrected to the start time of the measurement, and the corresponding activity value was then decayed to the time-point of the blood sampling.

\section{Evaluation of DNA damage: $\uparrow-\mathrm{H} 2 \mathrm{AX}+53 \mathrm{BP} 1$ assay}

The blood samples were processed and the $\gamma-\mathrm{H} 2 \mathrm{AX}+53 \mathrm{BP} 1$ foci evaluated following the protocol described by Eberlein et al. [22]. Briefly, leucocytes were separated by density centrifugation in BD Vacutainer CPT tubes (BD, Heidelberg, Germany), washed, fixed in $70 \%$ ethanol and stored at $-20{ }^{\circ} \mathrm{C}$. For immunofluorescence staining and focus analysis [23], the samples were shipped to the Bundeswehr Institute of Radiobiology in Munich, Germany. In each sample, $\gamma$ $\mathrm{H} 2 \mathrm{AX}+53 \mathrm{BP} 1$ colocalizing foci in 100 cells were counted. In a small number of samples, up to 200 cells were counted. To determine the average number of radiation-induced foci (RIF) per cell, the number of individual baseline foci per cell was subtracted from the number of counted foci per cell in all samples taken after administration of the radiopharmaceutical.

\section{Measurement of whole-body retention}

External dose-rate measurements and whole-body gamma camera scans (e.cam; Siemens Healthineers, Erlangen, Germany) were performed to determine the whole-body activity retention. For the dose-rate measurements, a ceiling-mounted shielded survey meter (automess - Automation und Messtechnik GmbH, Ladenburg, Germany) fixed $2.5 \mathrm{~m}$ above the patient's bed was used. At least seven measurements were taken in each patient. On the first day, measurements were taken directly ( $3 \mathrm{~min}$ up to a maximum of $38 \mathrm{~min}$ ) after administration, prior to the first postadministration void or defaecation by the patient, and then approximately $2 \mathrm{~h}, 4 \mathrm{~h}$ and $7 \mathrm{~h}$ after administration, followed by three more measurements every $12 \mathrm{~h}$ on the subsequent days. All measurement data were normalised to the first initial measurement. The whole-body gamma camera scans were performed nominally $4 \mathrm{~h}, 24 \mathrm{~h}, 48 \mathrm{~h}$ and $96 \mathrm{~h}$ after administration. The last whole-body scan ( $96 \mathrm{~h}$ after administration) was missing in patients $\mathrm{P} 5, \mathrm{P} 8$ and $\mathrm{P} 12$. In patient $\mathrm{P}$, a whole-body scan 
approximately $68 \mathrm{~h}$ after administration was performed instead. Additionally, all patients underwent a SPECT/CT scan (Symbia T2 or Symbia Intevo Bold; Siemens Healthineers, Erlangen, Germany) either $24 \mathrm{~h}$ or $48 \mathrm{~h}$ after administration. To avoid the possible effects of the radiation from the CT scan on the focus count, blood samples were always taken before the SPECT/CT scan.

The count rates in counts per second were evaluated in the anterior and the posterior image of each whole-body scan using syngo.via software (Siemens Healthineers, Erlangen, Germany). The geometric mean of the background-corrected anterior and posterior count rate was then calculated. The value for the first time-point of the external dose-rate measurement was decay-corrected to the time of administration and normalised to 1 , corresponding to $100 \%$ uptake. To combine the dose-rate measurements and the whole-body scan data, the count rates obtained from imaging performed $4 \mathrm{~h}$ after administration were normalised to the corresponding external doserate value $4 \mathrm{~h}$ after administration.

\section{Calculation of the absorbed doses to the blood using time-integrated activity coefficients}

Triexponential and biexponential fit functions were used to describe the time curves for the activity retention in the blood and in the whole-body, respectively, in each patient. By integrating the time-activity functions over time up to the timepoint $t$ of the respective blood withdrawal, time-integrated activity coefficients (TIACs) for the activity concentration in the blood $\left(\tau_{\mathrm{ml} \text { of bl }}(t)\right.$ in hours per millilitre) and for the wholebody $\left(\tau_{\mathrm{wb}}(t)\right.$ in hours) were obtained.

The absorbed doses to the blood $D_{\mathrm{bl}}(t)$ as a function of time were calculated as described by Eberlein et al. [14]. Additionally, the injection phase over the first $20 \mathrm{~min}$ with patient-specific TIAC values $\tau_{\mathrm{ml}}$ of bl,i for the blood contribution and $\tau_{\mathrm{wb}, \mathrm{i}}$ for the whole-body contribution was considered in this study, as the activity was administered using a syringe pump over $20 \mathrm{~min}$. The constant TIAC values $\tau_{\mathrm{ml}}$ of bl,i and $\tau_{\text {wb,i }}$ were calculated assuming a linear uptake for $-20 \mathrm{~min}<t$ $<0$ until the values of $\tau_{\mathrm{ml}}$ of bl $(t=0)$ and $\tau_{\mathrm{wb}}(t=0)$, respectively, were reached. This resulted in the following equation describing the absorbed dose to the blood:

$$
\begin{aligned}
D_{\mathrm{bl}}(t)=A_{0} & \cdot\left(85.3 \frac{\mathrm{Gy} \cdot \mathrm{ml}}{\mathrm{GBq} \cdot \mathrm{h}} \cdot\left(\tau_{\mathrm{ml} \text { of bl }}(t)+\tau_{\mathrm{ml} \text { of bl, i }}\right)\right. \\
+ & \left.\left(\frac{0.00185}{w t^{\frac{2}{3}}}\right) \frac{\mathrm{Gy} \cdot \mathrm{kg}^{\frac{2}{3}}}{\mathrm{GBq} \cdot \mathrm{h}} \cdot\left(\tau_{\mathrm{wb}}(t)+\tau_{\mathrm{wb}, \mathrm{i}}\right)\right)
\end{aligned}
$$

where $A_{0}$ is the administered activity in gigabecquerels and $w t$ is the weight of the patient in kilograms. A derivation of Eq. 1 and a detailed description of the constants therein is given in the Supplementary Material of reference [14].

The absorbed dose rate $\frac{\mathrm{d} D_{b l}}{\mathrm{~d} t}$ is defined as the derivative of the absorbed dose to the blood $D_{\mathrm{bl}}$ (Eq. 1) over time.

\section{Statistical analysis}

OriginPro 2017 (OriginLab Corporation) was used for data analysis, plotting and statistical evaluation. To test whether data were normally distributed, the Shapiro-Wilk test was used. For correlation analysis, Pearson correlation was applied for normally distributed datasets and Spearman correlation was applied if datasets were not normally distributed. Results were considered significant for $p<0.05$. To test for differences between two groups of normally distributed data, $t$-tests were used. Results were considered significant for Prob $>|t|<0.05$. The standard deviation of the focus count was calculated for each evaluated blood sample assuming a Poisson distribution of the counts in 100 cells. For the calculation of the standard deviation of the average number of RIF per cell, the standard deviation of the baseline focus count was considered additionally and error propagation was performed. Generally, the standard deviation is stated for all mean values. For the fit parameters, the standard error is given.

\section{Results}

\section{Patients}

A total of 16 heavily pretreated patients (P1-P16) aged between 54 and 81 years (average $70 \pm 9$ years) were enrolled. The mean administered activity was $5.9 \pm 0.2 \mathrm{GBq}$ of ${ }^{177} \mathrm{Lu}$. The demographic and clinical data of all patients are presented in Table 1.

The pretreatments included surgery (radical prostatectomy/ prostatovesiculectomy, lymphadenectomy, orchiectomy), external-beam radiation therapy, therapy with ${ }^{223} \mathrm{Ra}$ dichloride, therapy with LHRH agonists and antagonists (leuprorelide, degarelix), antiandrogen therapy with abiraterone, bicalutamide, enzalutamide and flutamide, chemotherapy (docetaxel, cabazitaxel) and therapy with denosumab. All patients had a diagnostic PET/CT scan with ${ }^{68}$ Ga-labelled PSMA 7 to 106 days before the start of therapy and again 39 to 114 days after therapy. Additionally, all patients underwent ${ }^{99 \mathrm{~m}}$ Tc-MAG3 scintigraphy 3 to 31 days before the start of therapy. Only in patients P2 and P4 was the MAG3 scan (with $<100 \mathrm{MBq}^{99 \mathrm{~m}} \mathrm{Tc}$ ) performed on the day of therapy, at least $3 \mathrm{~h}$ before administration of ${ }^{177} \mathrm{Lu}$-labelled PSMA. Of the 16 patients, 15 (all except P2) presented with 


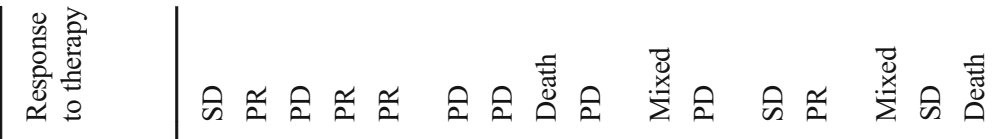

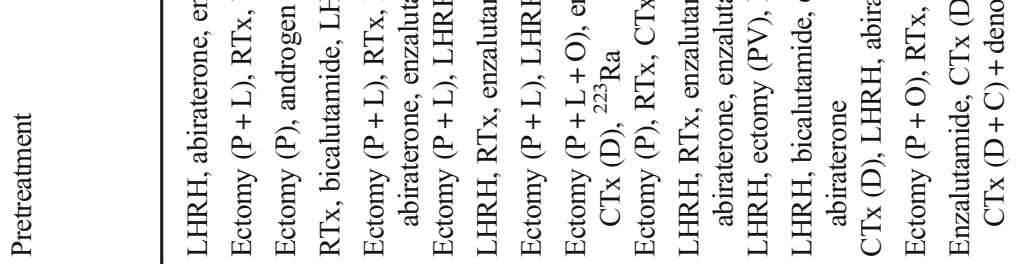

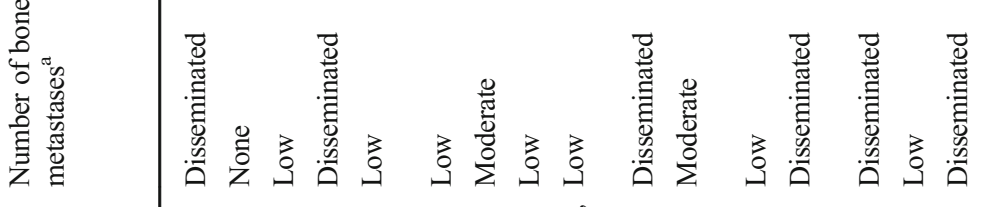<smiles>C1CCCCC1</smiles>

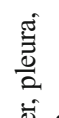

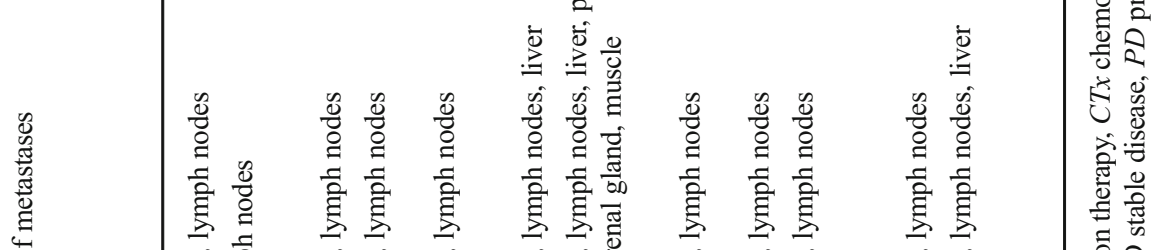

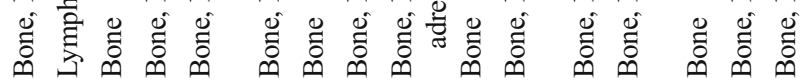

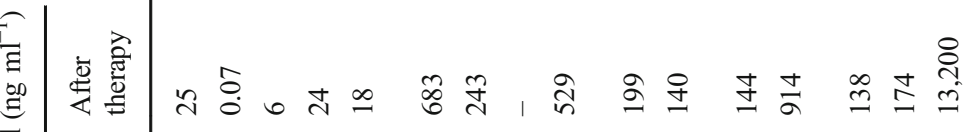

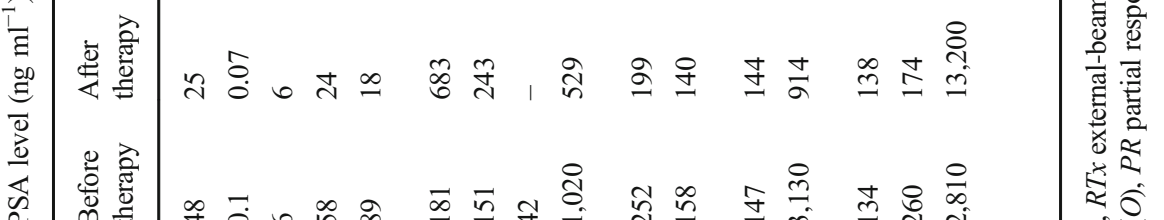

兽

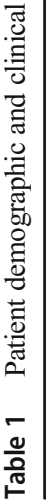

惫

$\frac{5}{500}$

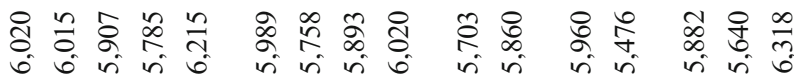

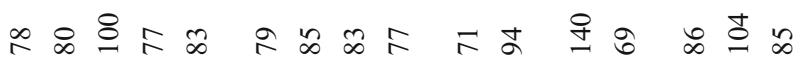

造造

-

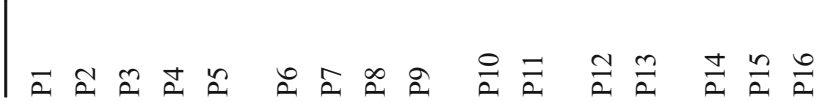


bone metastases and 12 (all except P3, P7, P10 and P14) presented with lymph node metastases. Three patients ( $\mathrm{P} 8$, P9 and P16) additionally had liver metastases. P9 also had metastases in the pleura, adrenal gland and muscles. After the first therapy cycle, four patients showed a partial response, three patients showed stable disease, five patients showed progressive disease, and two patients showed a mixed response. Two patients (P8 and P16) died after the first therapy cycle.

\section{Dosimetry}

A triexponential fit function was used to describe the activity retention in the blood. For describing the whole-body retention, a biexponential fit function sufficed to describe the kinetics as, in most patients, this resulted in the same curve but revealed smaller errors in the fit parameters than the triexponential fit function. With the corresponding TIAC values, which were obtained by integrating the time-activity functions over time up to the time-point $t$ of the respective blood withdrawal, the absorbed doses to the blood were calculated according to Eq. 1. Consequently, the absorbed doses represent cumulative doses from the start of administration to the respective time-point $t$.

The absorbed dose to the blood directly after administration (after the $20 \mathrm{~min}$ injection time) was $3.2 \pm 0.6 \mathrm{mGy}$ on average. The average total absorbed dose to the blood, which was calculated by integrating to $t=\infty$, was $109 \pm 28 \mathrm{mGy}$. The minimal total absorbed dose to the blood was 76 mGy (P3 and P11) and the maximal total absorbed dose to the blood was $164 \mathrm{mGy}$ (P9). The average absorbed doses to the blood at $t_{1}, t_{2}, t_{3}$ and $t_{4}$ were $17 \pm 6 \mathrm{mGy}, 27 \pm 7 \mathrm{mGy}, 34 \pm 8 \mathrm{mGy}$ and $40 \pm 9 \mathrm{mGy}$, respectively. For the later time-points, differences in the kinetics among the patients increased and values for the absorbed doses to the blood were no longer significantly drawn from a normally distributed population. The median absorbed doses to the blood at $t_{5}, t_{6}$ and $t_{7}$ were $71 \mathrm{mGy}$ ( $\mathrm{min}$ $52 \mathrm{mGy}$, max $129 \mathrm{mGy}$ ), $80 \mathrm{mGy}(\min 61 \mathrm{mGy}$, max $144 \mathrm{mGy}$ ) and $88 \mathrm{mGy}$ (min $69 \mathrm{mGy}$, max $155 \mathrm{mGy}$ ), respectively. At $t_{7}$, in all patients except $\mathrm{P} 9$, more than $80 \%$ of the total absorbed dose to the blood was reached. In patient P9, the absorbed dose to the blood at this time-point was only $56 \%$ of the calculated total absorbed dose to the blood.

Figure 1a shows the absorbed doses to the blood as a function of time in five selected patients. Patient P1 can be considered typical with a total absorbed dose to the blood of $103 \mathrm{mGy}$ and a characteristic time-dependent curve. Patient P8 showed a similar time-dependency of the absorbed dose to the blood. Patient P9 showed a much slower and longer increase in the absorbed dose to the blood as a function of time compared with that in most of the other patients. Patients P13 and P14 also showed similar time-dependency of the absorbed dose to the blood. Patients P10 and P11 showed the maximal
(155 mGy) and minimal (69 mGy) absorbed doses to the blood at the last sampling time-point, respectively.

The corresponding time-dependency of the absorbed dose rate $\frac{\mathrm{d} D_{\mathrm{bl}}}{\mathrm{d} t}$ is shown in Fig. $1 \mathrm{~b}$ in the same five patients. In all patients, the average absorbed dose rates at $t_{1}, t_{2}, t_{3}, t_{4}, t_{5}, t_{6}$ and $t_{7}$ were $11.59 \pm 2.98 \mathrm{mGy} \mathrm{h}^{-1}, 7.83 \pm 2.11 \mathrm{mGy} \mathrm{h}^{-1}, 6.03$ $\pm 1.85 \mathrm{mGy} \mathrm{h}^{-1}, 4.84 \pm 1.57 \mathrm{mGy} \mathrm{h}^{-1}, 0.72 \pm 0.26 \mathrm{mGy} \mathrm{h}^{-1}$, $0.29 \pm 0.14 \mathrm{mGy} \mathrm{h}^{-1}$ and $0.14 \pm 0.10 \mathrm{mGy} \mathrm{h}^{-1}$, respectively. At the last sampling time-point $t_{7}$, the absorbed dose rate was below $0.20 \mathrm{mGy} \mathrm{h}^{-1}$ in all patients except P9 $\left(0.41 \mathrm{mGy} \mathrm{h}^{-1}\right)$, P13 (0.26 mGy h $\left.{ }^{-1}\right)$ and P14 $\left(0.25 \mathrm{mGy} \mathrm{h}^{-1}\right)$.

\section{DNA damage foci}

The average number of foci per cell at baseline was $0.32 \pm 0.11$. Paired sample $t$-tests showed that the number of foci per cell at baseline was significantly different from the values at all later time-points $t_{1}$ to $t_{7}$. The individual baseline value in each patient was subtracted from the number of foci for all time-points after administration to calculate the average number of RIF per cell. The average numbers of RIF per cell over all patients at $t_{1}$, $t_{2}, t_{3}, t_{4}, t_{5}, t_{6}$ and $t_{7}$ were $0.29 \pm 0.14,0.39 \pm 0.15,0.38 \pm 0.09$, $0.38 \pm 0.17,0.27 \pm 0.13,0.21 \pm 0.14$ and $0.10 \pm 0.13$, respectively. The individual data for all patients are shown in Fig. 2.

Generally, the average number of RIF increased or remained constant from $t_{1}$ to $t_{4}$. This increase in the average number of RIF was significant from time-point $t_{1}$ to $t_{2}$, but not from $t_{2}$ to $t_{3}$ or from $t_{3}$ to $t_{4}$. Only in one patient (P8) was a continuous decrease in the average number of RIF observed during the first $4 \mathrm{~h}$ after administration, starting directly after the first time-point with $0.38 \pm 0.12 \mathrm{RIF}$ per cell to $0.03 \pm 0.10$ RIF per cell at $t_{4}$ (Fig. 1c). Starting from time-point $t_{4}$, the average number of RIF significantly decreased at all consecutive time-points. At the last time-point $t_{7}, 96 \mathrm{~h}$ after administration, in seven of 13 patients (P1, P2, P3, P4, P6, P11 and $\mathrm{P} 15)$ the average number of foci per cell had decreased to the individual baseline values, while in six patients (P7, P9, P10, P13, P14 and P16) the number of foci was still elevated. In three patients (P7, P9 and P14), the number of RIF per cell was still above 0.2 at this time-point. As an example, the average number of RIF per cell as a function of time in P9, the patient with the highest value $(0.38 \pm 0.10)$ at $t_{7}$, are shown in Fig. 1c (blue diamonds).

\section{Dose-dependency of the number of RIF per cell}

Figure 3 shows the average number of RIF per cell as a function of the absorbed dose to the blood in all 16 patients. A linear dependency of the absorbed dose to the blood $D_{\mathrm{bl}}$ and the average number of RIF per cell was assumed for the first three time-points $\left(t_{0}, t_{1}\right.$ and $t_{2}$; up to $2.6 \mathrm{~h}$ after administration). The corresponding dose range is shown in detail in Fig. $3 \mathrm{~b}$. 

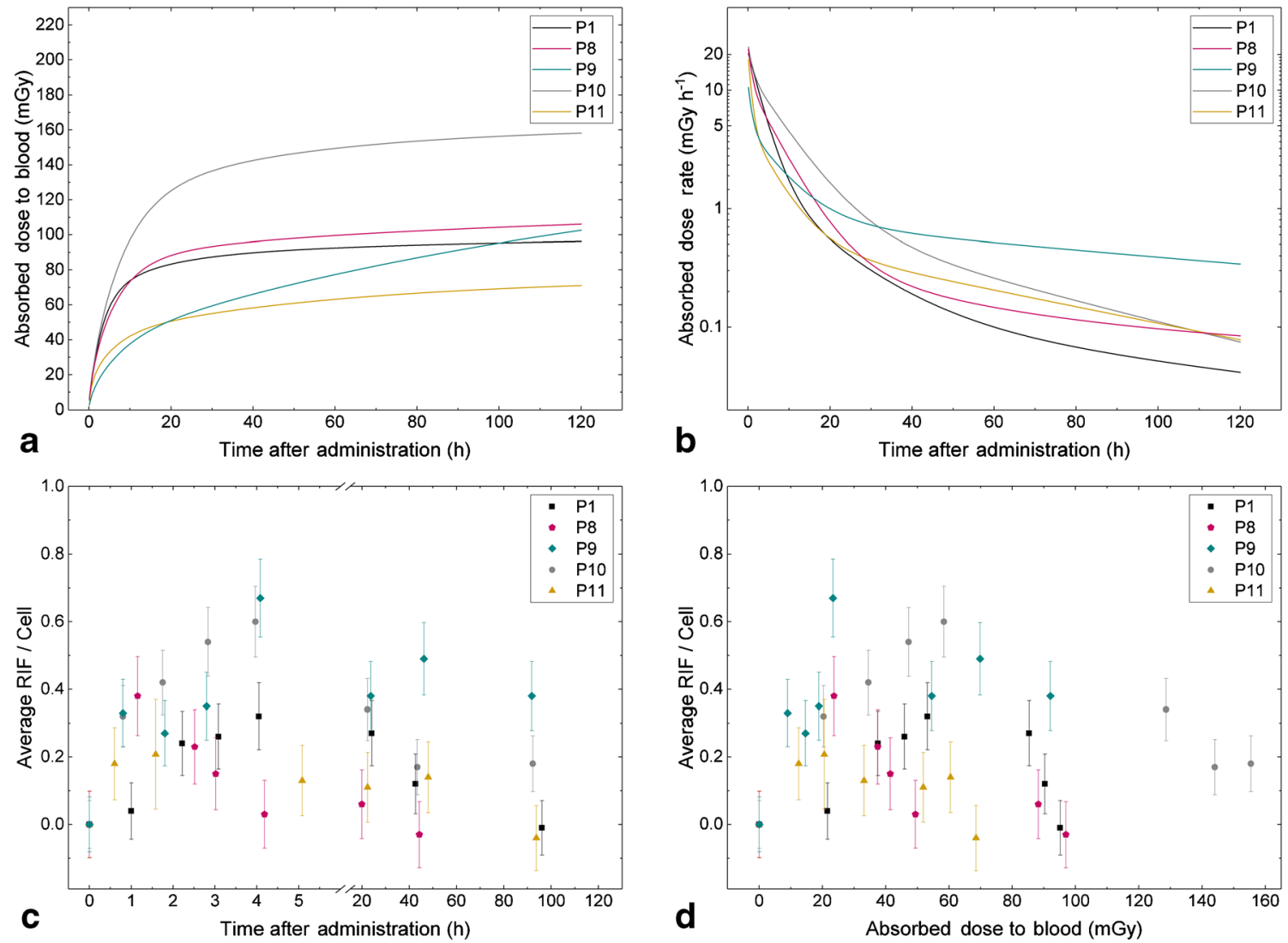

Fig. 1 Data of five selected patients: P1 with average parameters; P8, the only patient with a decrease in radiation-induced foci (RIF) directly after the first time-point; P9, the patient with the highest dose rate at the last time-point; P10, the patient with the highest absorbed dose to the blood at the last timepoint; and P11, the patient with the lowest absorbed dose to the blood at the last time-point. a Absorbed dose to the blood as a function of time. $\mathbf{b}$ Absorbed dose rate as a function of time. $\mathbf{c}$ Average number of RIF per cell as function of time (for better clarity a break is inserted in the $x$ axis). $\mathbf{d}$ Average number of RIF per cell as function of the absorbed dose to the blood

The patient data for these time-points were pooled and a linear fit to the pooled data was performed $\left(R^{2}=0.583\right)$ resulting in the linear equation:

\section{Average RIF per Cell}

$$
=(0.0336 \pm 0.0258)+(0.0122 \pm 0.0015) \mathrm{mGy}^{-1} \cdot D_{\mathrm{bl}}
$$
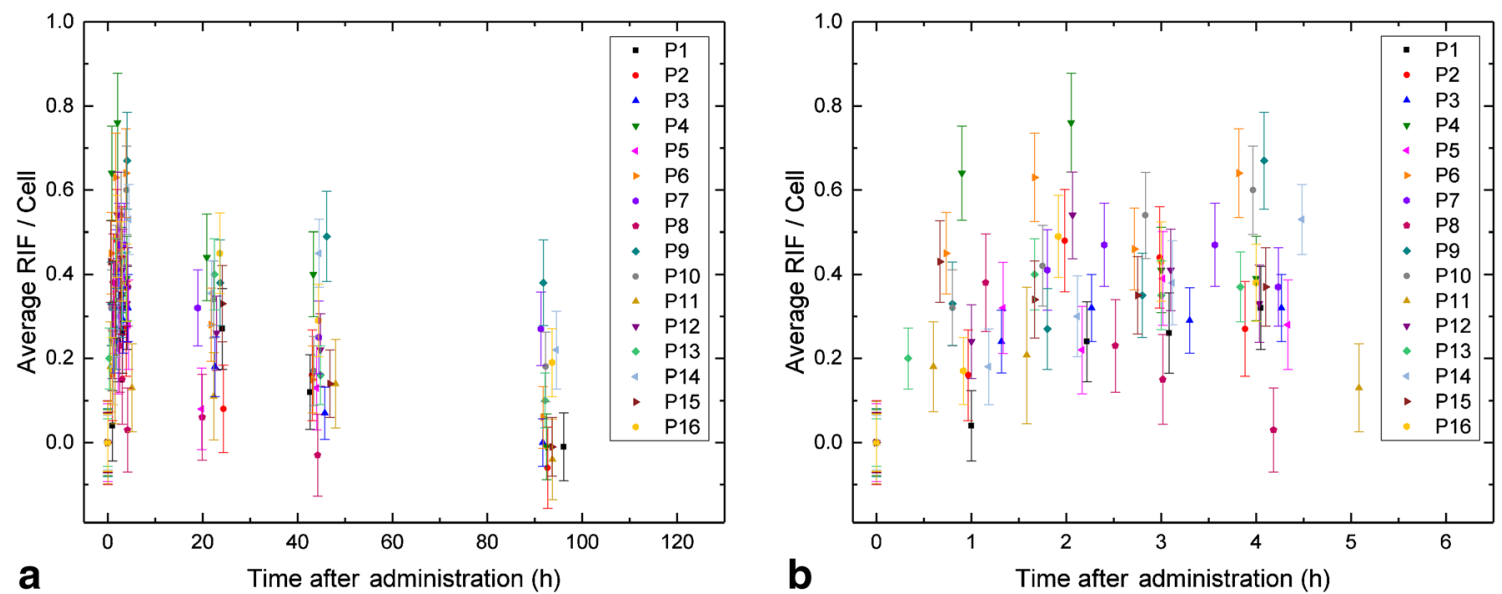

Fig. 2 Average number of RIF per cell as a function of time after administration of ${ }^{177}$ Lu-PSMA. a Overview of all time-points. $\mathbf{b}$ Detailed view of the first five time-points (nominally 0 to $4 \mathrm{~h}$ after administration) 

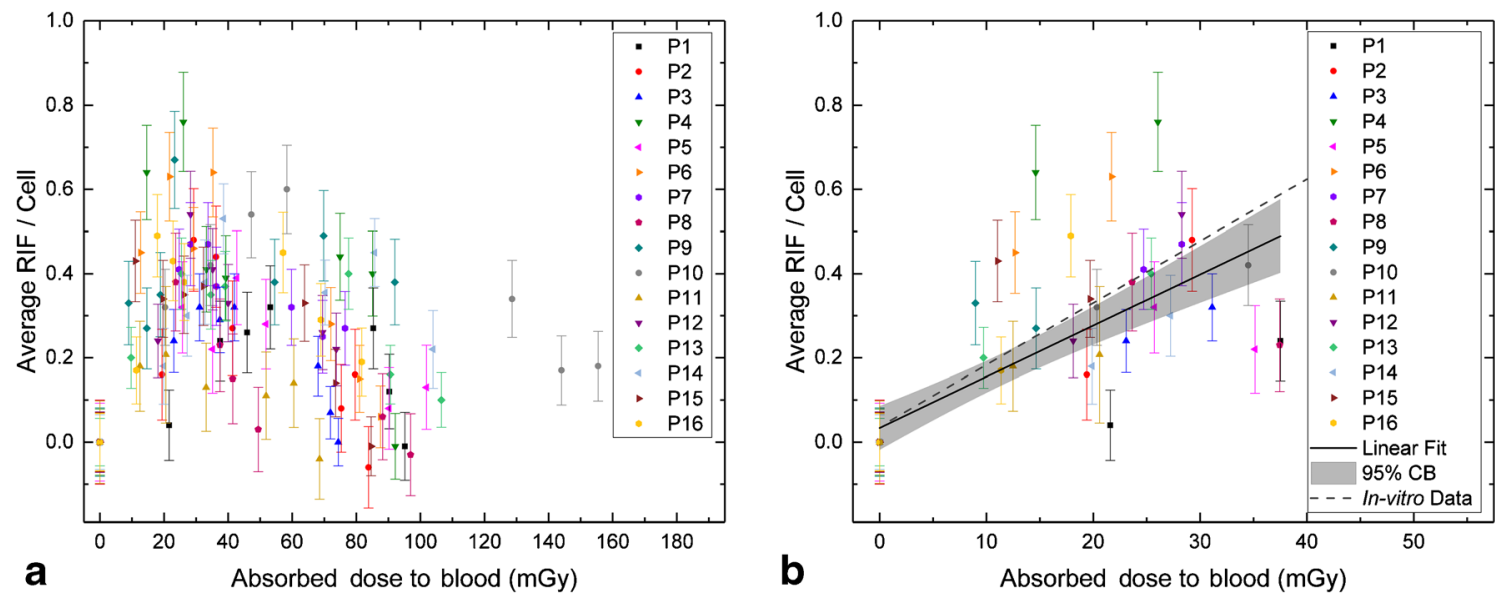

Fig. 3 Average number of RIF per cell as a function of the absorbed dose to the blood. a Overview of all time-points. $\mathbf{b}$ Detailed view of the first three time-points (up to $2.6 \mathrm{~h}$ after administration) with a linear fit (solid line) to the pooled data, including a 95\% confidence band (grey area). For comparison, the in-vitro calibration curve for ${ }^{177} \mathrm{Lu}$ and ${ }^{131} \mathrm{I}$ taken from reference [22] is also shown (dashed line)

The slope is significantly different from zero $(t=8.011$; Prob $>|t|=2.846 \mathrm{E}-10)$. The parameters for the lower (LCL) and upper (UCL) $95 \%$ confidence intervals are $\mathrm{LCL}=0.009$ and $\mathrm{UCL}=0.015$ for the slope and $\mathrm{LCL}=-0.018$ and $\mathrm{UCL}=$ 0.086 for the intercept. The slope obtained in this work is $17 \%$ smaller than the slope of an in-vitro calibration curve established for ${ }^{177} \mathrm{Lu}$ and ${ }^{131} \mathrm{I}$ in a previous study [22]. Figure $3 \mathrm{~b}$ shows the linear in-vitro calibration curve as a dashed line for comparison.

\section{Dose rate-dependency of the number of RIF per cell}

Figure 4a shows the average number of RIF per cell as a function of the absorbed dose rate $\frac{\mathrm{d} D_{\mathrm{bl}}}{\mathrm{d} t}$ in all 16 patients. The average numbers of RIF per cell for the last two sampling time-points $t_{6}$ and $t_{7}$ (with absorbed dose rates of less than $0.6 \mathrm{mGy} \mathrm{h}^{-1}$ ) are shown for 13 patients in more detail in Fig. 4b. The three patients with a missing $t_{7}$ value (P5, P8 and P12) were excluded from this graph and analysis. A fit to the pooled data revealed a linear relationship $\left(R^{2}=0.560\right)$ between the average number of RIF per cell and the absorbed dose rate in this range. The corresponding linear equation is:

Average RIF per Cell

$$
=(-0.0066 \pm 0.0326)+(0.6895 \pm 0.1248) \mathrm{h} \cdot \mathrm{mGy}^{-1} \cdot \frac{\mathrm{d} D_{\mathrm{bl}}}{\mathrm{d} t}
$$

The slope is significantly different from zero $(t=5.525$; Prob $>|t|=1.107 \mathrm{E}-5)$. The parameters for the $95 \%$ confidence intervals are $\mathrm{LCL}=0.432$ and $\mathrm{UCL}=0.947$ for the slope and $\mathrm{LCL}=-0.074$ and $\mathrm{UCL}=0.061$ for the intercept. In accordance with this result, there was a significant correlation between the average number of RIF per cell and the absorbed dose rate at $t_{6}$ (Pearson's $r=0.642$; $p=0.007$ ) and at $t_{7}$ (Pearson's $r=0.705 ; p=0.007$ ).

\section{Correlations with clinical parameters}

To test whether the variability in progression of RIF and the temporal course of the absorbed dose to the blood among the patients is linked to clinical parameters, correlation analysis was performed. However, because only 16 patients were included in the study, our results are based on a small amount of data. In the 13 patients with data for the last time-point $t_{7}$, there was a significant positive correlation between the PSA level before therapy and the absorbed dose rate $96 \mathrm{~h}$ after administration (Spearman's $\rho=0.819 ; p=0.001$ ). A weaker but still significant correlation was noted between the PSA level after therapy and the absorbed dose rate $96 \mathrm{~h}$ after administration (Spearman's $\rho=0.659 ; p=0.014$ ) and between the PSA level after therapy and the average number of RIF per cell $96 \mathrm{~h}$ after administration (Spearman's $\rho=0.619 ; p=0.024$ ). No significant correlations were observed between the number of bone metastases and the absorbed dose rate or the average number of RIF at $t_{7}$. Parameters describing kidney function (creatinine and MAG3 before therapy) did not correlate with the absorbed dose rate or the average number of RIF at $t_{7}$.

\section{Discussion}

In the current study, we used the $\gamma-\mathrm{H} 2 \mathrm{AX}+53 \mathrm{BP} 1 \mathrm{DSB}$ focus assay to investigate the time- and dose-dependency of DNA DSB induction and repair in blood leucocytes of prostate cancer patients during their first therapy with ${ }^{177} \mathrm{Lu}$ PSMA. In general, an increase in the average number of RIF was observed in the first hours after administration of the 

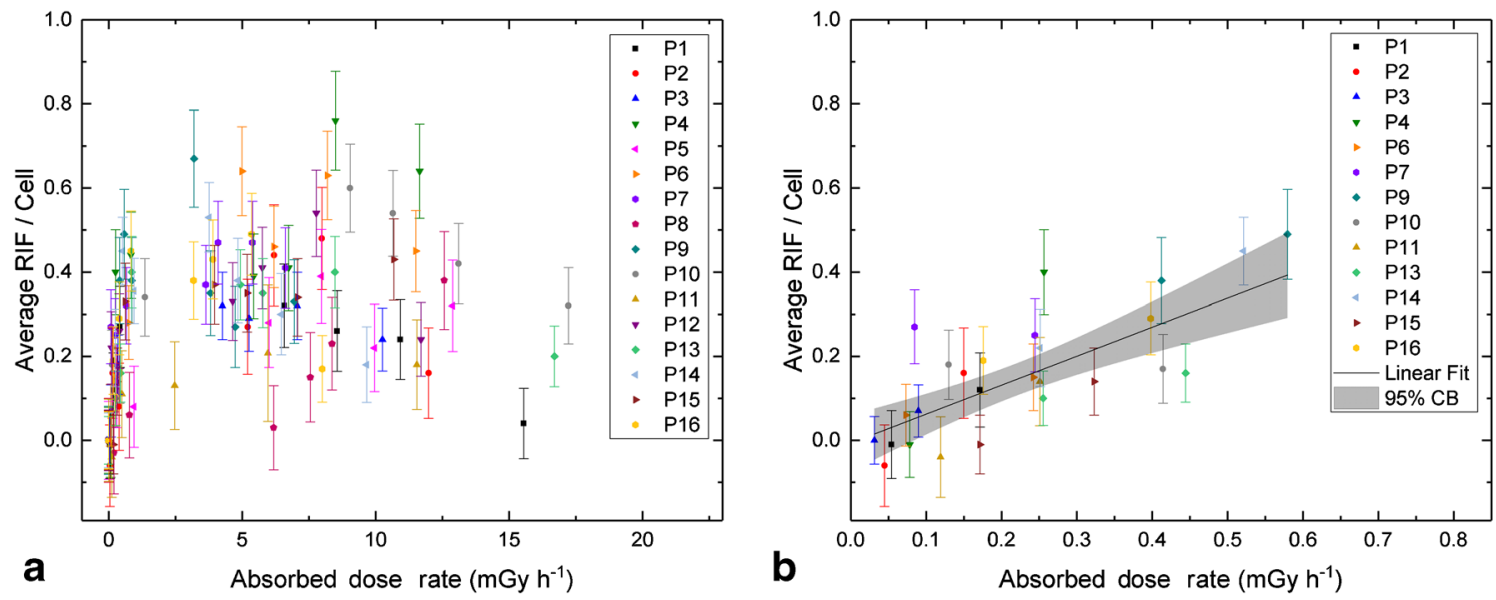

Fig. 4 Average number of RIF per cell as a function of the absorbed dose rate. a Overview of all time-points. b Detailed view of the last two time-points ( $48 \mathrm{~h}$ and $96 \mathrm{~h}$ after administration) with a linear fit (solid line) to the pooled data, including a $95 \%$ confidence band (grey area). Only the 13 patients with data for both time-points were included in the graph and in the fit

radiopharmaceutical, followed by a decrease due to DNA repair. A similar time course was also observed in previous studies investigating $\gamma-\mathrm{H} 2 \mathrm{AX}+53 \mathrm{BP} 1$ focus induction and repair after radioiodine therapy $[9,15]$ and peptide receptor radionuclide therapy with ${ }^{177} \mathrm{Lu}$-labelled DOTATATE/ DOTATOC [14]. In contrast to external irradiation, as in radiation therapy, internal exposure is characterized by continuous irradiation with a permanently decreasing dose rate after administration of the radionuclide. For this reason, a decrease in the number of RIF was observed at later time-points, despite the increasing absorbed dose to the blood. The disappearance of RIF at later time-points could also have been caused potentially by elimination of damaged cells. However, while high dose rate acute photon irradiation has been shown to induce apoptotic cell death in blood samples irradiated ex vivo [24, $25]$ and in minipigs exposed to partial body $\gamma$-irradiation with 50 Gy [23], we only occasionally noted apoptotic leucocytes in our in-vivo samples exposed to low doses and low dose rates. Therefore, we consider it unlikely that a preferential elimination of damaged cells influenced the repair time courses observed.

In the present study, the mean total absorbed dose to the blood was $109 \pm 29 \mathrm{mGy}$ after administration of $5.9 \pm$ $0.2 \mathrm{GBq}$. The median absorbed dose to the blood $48 \mathrm{~h}$ after administration was $80 \mathrm{mGy}$ ( $\min 61 \mathrm{mGy}$; max $144 \mathrm{mGy}$ ). This is comparable with the results of Eberlein et al. who observed an absorbed dose to the blood of $79 \pm 16 \mathrm{mGy}$ $48 \mathrm{~h}$ after administration of $7.2 \pm 0.4 \mathrm{GBq}{ }^{177} \mathrm{Lu}$-labelled DOTATATE/DOTATOC [14]. The total absorbed dose to the blood could not be calculated by Eberlein et al. since no data were available for time-points $>48 \mathrm{~h}$ after administration [14].

Up to $2.6 \mathrm{~h}$ after administration (time-points $t_{0}, t_{1}$ and $t_{2}$ ), there was a linear relationship between the average number of RIF per cell and the absorbed dose to the blood. A linear increase in the average number of RIF was also observed in the first $2 \mathrm{~h}$ after administration of ${ }^{131} \mathrm{I}[15]$ and up to $5 \mathrm{~h}$ after administration of ${ }^{177} \mathrm{Lu}$-DOTATATE/DOTATOC [14]. As in these previous studies, the slope of the linear curve obtained in this investigation was less than the slope of the in-vitro calibration curve established for ${ }^{177} \mathrm{Lu}$ and ${ }^{131} \mathrm{I}$ by Eberlein et al. [22]. Here, the difference to the in-vitro slopes was $17 \%$, while in the patient studies investigating the induction of RIF after radioiodine therapy and therapy with ${ }^{177}$ Lu-DOTATATE/ DOTATOC, the differences between the slopes of the invivo and the in-vitro data were $20 \%$ and $14 \%$, respectively $[14,15]$. The slightly shallower slope observed in all patient studies can be explained by the onset of DNA repair. Another reason for the differences between the slopes may be the greater inaccuracy in the calculation of the absorbed doses for the early time-points, as the first measurement for activity quantification was taken not earlier than $1 \mathrm{~h}$ after administration. Finally, it is possible that the difference in cellular environment (redox state) between the systemic in-vivo exposure and the "artificial" ex-vivo irradiation in blood tubes may have led to a lower induction of DSBs in patients.

While the average number of RIF per cell correlated with the absorbed dose to the blood during the first hours after administration of the radiopharmaceutical, we found that the absorbed dose rate was the determining factor for RIF progression at later time-points $t_{6}$ and $t_{7}(48 \mathrm{~h}$ and $96 \mathrm{~h}$ after administration). During the first hours after administration, no correlation was observed between the absorbed dose rate and the average number of RIF per cell. For the late timepoints $t_{6}$ and $t_{7}$, however, there was a linear relationship between the dose rate and the average number of RIF per cell. This indicates that patients with relatively high absorbed dose rates exhibit either slower focus repair kinetics or an increased rate of focus induction even at late time-points. An absorbed dose rate-dependency of RIF was also observed by Lassmann et al. in patients with differentiated thyroid cancer undergoing 
radioiodine therapy [9]. In other studies investigating $\gamma$ $\mathrm{H} 2 \mathrm{AX}$ foci after radionuclide therapy $[14,15,17,18]$, an absorbed dose rate-dependency of RIF was not considered.

For the latest time-points in particular, we also observed a high variability in the data among the patients. In seven of 13 patients, the number of foci per cell $96 \mathrm{~h}$ after administration had decreased to the baseline values. The other patients still showed elevated numbers of foci correlating with higher absorbed dose rates at this time-point.

To find a link between medical parameters and the variable effectiveness of DNA DSB repair among the patients, we compared the RIF and dose rate data with clinical endpoints and found significant correlations with PSA levels. However, for the correlation analysis only the data from 13 patients with different pretreatments could be included. To validate the current findings and to exclude possible confounding factors, more patients and data are needed.

Although the general pattern of the time- and dosedependencies of RIF induction were similar to those found in previous studies $[14,15]$, we observed a higher variability among the patients overall in this study, which is reflected in a wider distribution of the foci data and a weaker correlation for the linear fit as a function of the absorbed dose to the blood for the first data points. A possible reason for this could be the comparatively longer medical history and the individual variability in the extensive pretreatments of the patients. Other possible causes of the variability among the patients are variations in the absorbed dose delivery directly after administration and differences in individual repair kinetics. In particular at late time-points, repair kinetics are influenced by varying dose rates. In order to link the dose-dependency in the first hours after administration and the dose rate-dependency for the last sampling time-points, patient-specific kinetic modelling, taking into account the dose-dependency of focus induction and repair, may be applied in future studies.

\section{Conclusion}

This is the first study of the induction and persistence of DSB DNA damage combined with internal dosimetry after therapy with ${ }^{177} \mathrm{Lu}$-PSMA. The general pattern of the time-dependent induction and disappearance of RIF follows that of other radionuclide therapies. In some patients, RIF had not disappeared completely $96 \mathrm{~h}$ after administration, which can be explained by an above average absorbed dose rate. In the majority of patients, however, the DSBs induced in blood leucocytes were effectively repaired. The correlation with clinical findings needs further research in a larger number of patients.
Acknowledgements We thank all patients for their participation in the study. We also thank the members of the Department of Nuclear Medicine and the Bundeswehr Institute of Radiobiology for support and assistance, in particular Cornelia Schubert, Mona Laubmeier and Jessica Müller.

Authors' contributions U.E., S.Sc., M.L. and H.S. designed the study and developed the methodology. C.L. and S.Se. recruited the patients, performed the blood sampling and acquired medical data. S.Sc., U.E. and M.L. processed the blood samples and performed the calculations of the absorbed doses. H.S. and R.M. performed $\gamma$-H2AX + 53BP1 staining and analysis. S.Sc., H.S., U.E. and M.L. analysed and interpreted the data and wrote the manuscript. All authors reviewed the manuscript. All authors read and approved the final manuscript.

Funding This study was funded by a grant of the Deutsche Forschungsgemeinschaft (DFG; grant nos. LA 2304/3-2 and SCHE $350 / 12-2$ ). The funders had no role in study design, data collection, analysis and interpretation, decision to publish, or preparation of the manuscript.

\section{Compliance with ethical standards}

Conflicts of interest Michael Lassmann has received research grants from Siemens Healthcare, Ipsen Pharma, Nordic Nanovector, ABX advanced biochemical compounds and is a member of the board of the EANM. Uta Eberlein is member of the EANM Dosimetry Committee. The other authors declare that they have no conflicts of interest.

Ethics approval The research plan was presented to the Ethics Committee of the Medical Faculty of the University of Würzburg, Germany (Az: 209/14). The Ethics Committee approved the study stating that there were no objections to the conduct of the study. All procedures performed in studies involving human participants were in accordance with the ethical standards of the Ethics Committee of the Medical Faculty of the University of Würzburg and with the principles of the 1964 Declaration of Helsinki and its later amendments or comparable ethical standards.

Informed consent Informed consent was obtained from all individual participants included in the study.

Open Access This article is distributed under the terms of the Creative Commons Attribution 4.0 International License (http:// creativecommons.org/licenses/by/4.0/), which permits unrestricted use, distribution, and reproduction in any medium, provided you give appropriate credit to the original author(s) and the source, provide a link to the Creative Commons license, and indicate if changes were made.

\section{References}

1. Ghosh A, Heston WD. Tumor target prostate specific membrane antigen (PSMA) and its regulation in prostate cancer. J Cell Biochem. 2004;91(3):528-39. https://doi.org/10.1002/jcb.10661.

2. Sweat SD, Pacelli A, Murphy GP, Bostwick DG. Prostate-specific membrane antigen expression is greatest in prostate adenocarcinoma and lymph node metastases. Urology. 1998;52(4):637-40.

3. Ahmadzadehfar H, Rahbar K, Kurpig S, Bogemann M, Claesener M, Eppard E, et al. Early side effects and first results of radioligand therapy with (177)Lu-DKFZ-617 PSMA of castrate-resistant metastatic prostate cancer: a two-centre study. EJNMMI Res. 2015;5: 36. https://doi.org/10.1186/s13550-015-0114-2. 
4. Rahbar K, Ahmadzadehfar H, Kratochwil C, Haberkorn U, Schafers M, Essler M, et al. German multicenter study investigating 177Lu-PSMA-617 radioligand therapy in advanced prostate cancer patients. J Nucl Med. 2017;58(1):85-90. https://doi.org/10.2967/ jnumed.116.183194.

5. Hofman MS, Violet J, Hicks RJ, Ferdinandus J, Thang SP, Akhurst T, et al. [(177)Lu]-PSMA-617 radionuclide treatment in patients with metastatic castration-resistant prostate cancer (LuPSMA trial): a single-centre, single-arm, phase 2 study. Lancet Oncol. 2018;19(6):825-33. https://doi.org/10.1016/S1470-2045(18) 30198-0.

6. Kratochwil C, Giesel FL, Stefanova M, Benesova M, Bronzel M, Afshar-Oromieh A, et al. PSMA-targeted radionuclide therapy of metastatic castration-resistant prostate cancer with $177 \mathrm{Lu}$-labeled PSMA-617. J Nucl Med. 2016;57(8):1170-6. https://doi.org/10. 2967/jnumed.115.171397.

7. Baum RP, Kulkarni HR, Schuchardt C, Singh A, Wirtz M, Wiessalla S, et al. 177Lu-labeled prostate-specific membrane antigen radioligand therapy of metastatic castration-resistant prostate cancer: safety and efficacy. J Nucl Med. 2016;57(7):1006-13. https://doi.org/10.2967/jnumed.115.168443.

8. Kulkarni HR, Singh A, Schuchardt C, Niepsch K, Sayeg M, Leshch $\mathrm{Y}$, et al. PSMA-based radioligand therapy for metastatic castrationresistant prostate cancer: the Bad Berka experience since 2013. J Nucl Med. 2016;57(Suppl 3):97S-104S. https://doi.org/10.2967/ jnumed.115.170167.

9. Lassmann M, Hänscheid H, Gassen D, Biko J, Meineke V, Reiners $\mathrm{C}$, et al. In vivo formation of gamma-H2AX and 53BP1 DNA repair foci in blood cells after radioiodine therapy of differentiated thyroid cancer. J Nucl Med. 2010;51(8):1318-25. https://doi.org/ 10.2967/jnumed.109.071357.

10. Rogakou EP, Boon C, Redon C, Bonner WM. Megabase chromatin domains involved in DNA double-strand breaks in vivo. J Cell Biol. 1999;146(5):905-16.

11. Rogakou EP, Pilch DR, Orr AH, Ivanova VS, Bonner WM. DNA double-stranded breaks induce histone H2AX phosphorylation on serine 139. J Biol Chem. 1998;273(10):5858-68.

12. Schultz LB, Chehab NH, Malikzay A, Halazonetis TD. p53 binding protein 1 (53BP1) is an early participant in the cellular response to DNA double-strand breaks. J Cell Biol. 2000;151(7):1381-90.

13. Chowdhury D, Keogh MC, Ishii H, Peterson CL, Buratowski S, Lieberman J. Gamma-H2AX dephosphorylation by protein phosphatase 2A facilitates DNA double-strand break repair. Mol Cell. 2005;20(5):801-9. https://doi.org/10.1016/j.molcel.2005.10.003.

14. Eberlein U, Nowak C, Bluemel C, Buck AK, Werner RA, Scherthan $\mathrm{H}$, et al. DNA damage in blood lymphocytes in patients after (177)Lu peptide receptor radionuclide therapy. Eur J Nucl Med Mol Imaging. 2015;42(11):1739-49. https://doi.org/10.1007/ s00259-015-3083-9.

15. Eberlein U, Scherthan H, Bluemel C, Peper M, Lapa C, Buck AK, et al. DNA damage in peripheral blood lymphocytes of thyroid cancer patients after radioiodine therapy. J Nucl Med. 2016;57(2): 173-9. https://doi.org/10.2967/jnumed.115.164814.

16. Rothkamm K, Löbrich M. Evidence for a lack of DNA doublestrand break repair in human cells exposed to very low x-ray doses. Proc Natl Acad Sci U S A. 2003;100(9):5057-62. https://doi.org/ 10.1073/pnas.0830918100.

17. Doai M, Watanabe N, Takahashi T, Taniguchi M, Tonami H, Iwabuchi K, et al. Sensitive immunodetection of radiotoxicity after iodine-131 therapy for thyroid cancer using gamma-H2AX foci of DNA damage in lymphocytes. Ann Nucl Med. 2013;27(3):233-8. https://doi.org/10.1007/s12149-012-0678-0.

18. Denoyer D, Lobachevsky P, Jackson P, Thompson M, Martin OA, Hicks RJ. Analysis of 177Lu-DOTA-octreotate therapy-induced DNA damage in peripheral blood lymphocytes of patients with neuroendocrine tumors. J Nucl Med. 2015;56(4):505-11. https:// doi.org/10.2967/jnumed.114.145581.

19. Eisenhauer EA, Therasse P, Bogaerts J, Schwartz LH, Sargent D, Ford R, et al. New response evaluation criteria in solid tumours: revised RECIST guideline (version 1.1). Eur J Cancer. 2009;45(2): 228-47. https://doi.org/10.1016/j.ejca.2008.10.026.

20. Wahl RL, Jacene H, Kasamon Y, Lodge MA. From RECIST to PERCIST: evolving considerations for PET response criteria in solid tumors. J Nucl Med. 2009;50(Suppl 1):122S-50S. https://doi. org/10.2967/jnumed.108.057307.

21. Scher HI, Morris MJ, Stadler WM, Higano C, Basch E, Fizazi K, et al. Trial design and objectives for castration-resistant prostate cancer: updated recommendations from the Prostate Cancer Clinical Trials Working Group 3. J Clin Oncol. 2016;34(12): 1402-18. https://doi.org/10.1200/JCO.2015.64.2702.

22. Eberlein U, Peper M, Fernandez M, Lassmann M, Scherthan H. Calibration of the gamma-H2AX DNA double strand break focus assay for internal radiation exposure of blood lymphocytes. PLoS One. 2015;10(4):e0123174. https://doi.org/10.1371/journal.pone. 0123174.

23. Lamkowski A, Forcheron F, Agay D, Ahmed EA, Drouet M, Meineke V, et al. DNA damage focus analysis in blood samples of minipigs reveals acute partial body irradiation. PLoS One. 2014;9(2):e87458. https://doi.org/10.1371/journal.pone.0087458.

24. Wilkins RC, Wilkinson D, Maharaj HP, Bellier PV, Cybulski MB, McLean JR. Differential apoptotic response to ionising radiation in subpopulations of human white blood cells. Mutat Res. 2002;513(1-2):27-36.

25. Horn S, Barnard S, Brady D, Prise KM, Rothkamm K. Combined analysis of gamma-H2AX/53BP1 foci and caspase activation in lymphocyte subsets detects recent and more remote radiation exposures. Radiat Res. 2013;180(6):603-9. https://doi.org/10.1667/ RR13342.1.

Publisher's note Springer Nature remains neutral with regard to jurisdictional claims in published maps and institutional affiliations. 